\title{
Isolation of filamentous fungi exhibiting high endoxylanase activity in lignocellulose hydrolysate
}

\author{
Christoph Ottenheim ${ }^{\mathrm{a}, \mathrm{b}}$, Kirstin Meier ${ }^{\mathrm{b}}$, Wolfgang Zimmermann ${ }^{\mathrm{a}}$, Jin Chuan Wu b*
}

\begin{abstract}
For complete degradation of hemicellulose into its monomers from lignocellulose biomass, the synergistic action of a broad range of hydrolytic enzymes is needed. Therefore, production of enzymes from their natural producer is desirable. To obtain a powerful $\beta$-1,4-endoxylanase producing fungus, 304 environmental samples were collected from various locations in Singapore, leading to 603 isolates. Among them, 71 exhibiting $\beta$-1,4-endoxylanase activity were identified belonging mainly to the genera of Aspergillus, Penicillium, and Trichoderma. Further analysis revealed Aspergillus niger DSM 26641 as a potential and stable $\beta$-1,4-endoxylanase producer, being able to grow in hydrothermal lignocellulose hydrolysate exhibiting its maximal $\beta$-1,4-endoxylanase activity at $\mathrm{pH} 4$ and $60^{\circ} \mathrm{C}$. This strain is thought to be very suitable for lactic acid production in a simultaneous saccharification and fermentation at pH values below 5 .

\section{Keywords}

$\beta$-1,4-endoxylanase, On-site enzyme production, Filamentous fungi, Lignocellulose, Aspergillus niger, Phylogeny

a Microbiology and Bioprocess Engineering, Institute of Biochemistry, Leipzig University, 04103 Leipzig, Germany

b Industrial Biotechnology, A*STAR Institute of Chemical and Engineering Sciences, 1 Pesek Road, Jurong Island, Singapore 627833

Email adresses: christoph@ottenheim.net, kmeier@uni-bonn.de, wolfgang.zimmermann@uni-leipzig.de, wu_jinchuan@ices.a-star.edu.sg

*Corresponding author: Dr. Wu jin Chuan, wu_jinchuan@ices.a-star.edu.sg, Tel: +65-67963803; Fax: +65-63166182
\end{abstract}

\section{Preface for this PDF version}

This article is a derivate of the accepted research article published in Applied Biochemistry and Biotechnology in Vol. 175:4, 2066-2074 in 2015 through Springer. The original article is available under 10.1007/s12010-0141427-8. This pdf version is not citable and not meant for commercial use. Every use of this PDF others than for research purpose is not allowed. The storage of this PDF is only allowed on the first authors private webpage (www.ottenheim.net). A collection in repositories is not approvable. Please link to the article but do not offer downloads of this article. All material in this PDF remain under the original copyright of the article published through Springer. For any issues arising from this PDF please contact christoph@ottenheim.net.

\section{Introduction}

Lignocellulose, the widely abundant biomass, is mainly composed of cellulose (20-50\%), hemicellulose (15-35\%) and lignin (15-35\%) [1, 2]. It is seen as a promising resource for production of value-added chemicals such as ethanol and lactic acid [3]. Degradation of the sugar polymers to their monomers is essential for microbial fermentation. The complete degradation of hemicellulose needs the synergistic action of a group of hydrolytic enzymes [4]. Even though these enzymes can be produced in larger quantities individually by recombinant DNA technology $[5,6,7,8]$, full and fast degradation of hemicellulose can only be achieved by the synergistic action of all these enzymes [9]. The compositions of hemicellulose differ between plants and even between different parts of a single plant. Therefore, a process based on whole cell enzyme production would provide flexibility for efficient enzymatic hydrolysis of hemicellulose.

Filamentous fungi are natural degraders of lignocellu- 
lose and therefore also potential producers of complex enzyme mixtures. Their growth occurs naturally on solid substrates but they are also able to grow in submerged cultures forming various morphological structures like pellets which are tightly associated with enzyme production [10]. As submerged fermentation has well established protocols, it is favored industrially over solid state fermentation which is often more difficult to control.

In general, the usability of an enzyme mixture is dependent on its cost-effectiveness as the success of production of bulk chemicals is cost-driven. Process costs need to be reduced by measures such as on-site enzyme production [11], recycling of fungal pellets and avoidance of genetic modifications which lowers wastewater treatment costs. On the other hand, the selected fungal strain is of uttermost importance. In addition to a high production of key enzymes like $\beta$-1, 4-endoxylanases which are responsible for hydrolysis of xylan, the major component of hemicellulose, the enzymes need to have suitable $\mathrm{pH}$ and temperature optima for the intended applications.

Here we report the isolation of a filamentous fungus Aspergillus niger DSM 26641 from the Singaporean environment exhibiting high $\beta$-1, 4-endoxylanase activity in submerged culture of lignocellulose hydrolysate with desired $\mathrm{pH}$ and temperature optima for simultaneous saccharification and fermentation of lignocellulose.

\section{Materials and Methods}

\subsection{Sample collection, strain isolation and storage}

For obtaining filamentous fungi secreting $\beta$-1, 4-endoxylanases, samples were collected from the Singaporean environment and processed depending on their origins. Solid samples like soil, plant parts and rotten materials were submerged, vigorously mixed and diluted with autoclaved water while liquid samples were directly diluted. Fungal fruiting bodies were partially sterilized by submersion in technical ethanol. Subsequently, the treated samples were transferred to primary pre-dried PDA plates (potato extract $4 \mathrm{~g} \mathrm{l}^{-1}$, dextrose $20 \mathrm{~g} \mathrm{l}^{-1}$, agar $20 \mathrm{~g} \mathrm{l}^{-1}, 50 \mathrm{mg} \mathrm{l}^{-1}$ chloramphenicol dissolved in methanol). The addition of chloramphenicol excluded the growth of bacteria while the extensive drying of the agar plates before usage made it unlikely for yeasts to emerge. After incubation of the primary PDA plates for $4-5$ days at $30^{\circ} \mathrm{C}$, single colonies of morphologically unique filamentous fungi were repeatedly transferred to fresh secondary PDA plates until morphological uniformity was observed. For mid-term storage, the obtained isolates were stored at $4{ }^{\circ} \mathrm{C}$ and transferred to new PDA plates every 6 months. For longterm storage, fungal mycelium and spores from fresh PDA plates were stored in $20 \%$ glycerol at $-80^{\circ} \mathrm{C}$.

\subsection{Screening for $\boldsymbol{\beta}-1$, 4-endoxylanase activity}

For $\beta$-1, 4-endoxylanase activity screening, enzymes were produced in submerged cultures using a defined broth (beechwood xylan $2 \mathrm{~g} \mathrm{l}^{-1}$, yeast extract $0.1 \mathrm{~g} \mathrm{l}^{-1}$, peptone $0.1 \mathrm{~g} \mathrm{l}^{-1}, 0.1 \mathrm{~g} \mathrm{l}^{-1}$ xylose). Broth $(5 \mathrm{ml})$ in $15 \mathrm{ml}$ tubes were inoculated with spores or mycelium from the prior obtained isolates and incubated horizontally for 4 days at $30^{\circ} \mathrm{C}$ and $200 \mathrm{rpm}$. Subsequently, tubes were centrifuged to separate mycelium and culture supernatant. Remazol Brilliant Blue R dyed xylan (RBB-Xylan) prepared following an earlier developed protocol [12] was used to determine $\beta$-1, 4-endoxylanase activity in the obtained culture supernatants according to a modified protocol [13]. The absorbance was measured at $590 \mathrm{~nm}$ and used directly or set as a percentage to the highest measured absorbance value during the respective screening. Due to the initial large amount of fungal isolates, the first screening of all isolates was done in batches to exclude isolates which did not significantly produce $\beta-1$, 4-endoxylanase.

\subsection{Identification and phylogenetic classification}

Fungal mycelium of isolates identified as potential $\beta$-1, 4-endoxylanase producers was grown in $15 \mathrm{ml}$ tubes as described earlier. After centrifugation, the mycelium was washed with autoclaved water to remove possibly formed spores and residual culture supernatant. The mycelium was transferred into $2 \mathrm{ml}$ thick-walled reaction tubes and a ceramic ball with $100 \mathrm{mg}$ quartz sand (50-70 mesh) was added. Additionally, $1 \mathrm{ml}$ extraction buffer (SDS $5 \mathrm{~g} \mathrm{l}^{-1}$, EDTA $7.3 \mathrm{~g} \mathrm{l}^{-1}$, NaCl $14.61 \mathrm{~g} \mathrm{l}^{-1}$, TRIS $24.23 \mathrm{~g} \mathrm{l}^{-1}, \mathrm{pH}$ 8.5) was supplied. The prepared samples were homogenized at $4.5 \mathrm{~m} \mathrm{~s}^{-1}$ for $20 \mathrm{~s}$ using the FastPrep24-Homogenizer (MP Biomedicals) and subsequently centrifuged for $10 \mathrm{~min}$ at $20,800 \mathrm{x} \mathrm{g}$ at $4^{\circ} \mathrm{C}$ to remove non-homogenized biomass. The supernatants were transferred into new reaction tubes and phenol : chloroform : isoamylalcohol $(1 \mathrm{ml})$ was added. After 
vigorous mixing, the samples were centrifuged for $15 \mathrm{~min}$ at $20,800 \mathrm{x} \mathrm{g}$ and $4^{\circ} \mathrm{C}$. The aqueous phases $(0.5 \mathrm{ml})$ were transferred to new $1.5 \mathrm{ml}$ reaction tubes. Ethanol $(1 \mathrm{ml}, 100 \%)$ and sodium acetate $(0.15 \mathrm{ml}, 3 \mathrm{M})$ was added followed by vigorous mixing. After precipitation overnight at $-80^{\circ} \mathrm{C}$, samples were centrifuged at

$20,800 \mathrm{x} g$ for $30 \mathrm{~min}$ at $4^{\circ} \mathrm{C}$. The supernatants were discarded and pellets were washed twice with $70 \%(\mathrm{v} / \mathrm{v})$ ethanol. Subsequently, pellets were air-dried and dissolved in $50 \mu \mathrm{l}$ DNAse free water. Nucleic acid concentrations were estimated using a Nanodrop 2000 spectrophotometer.

For amplification by Polymerase Chain Reaction (PCR), a mixture containing $5 \mu \mathrm{l}$ Taq buffer (10X), $3 \mu \mathrm{l}$ $\mathrm{MgCl}_{2}(25 \mathrm{mM}), 1 \mu \mathrm{l}$ dNTP Mix (10 mM), $1 \mu \mathrm{l}$ primer ITS1 (10 mM) (5'- TCC GTA GGT GAA CCT GCG G-3'), $1 \mu$ l primer ITS4 (10 mM) (5‘- TCC TCC GCT TAT TGA TAT GC-3') and $0.3 \mu$ l Taq polymerase $(5 \mathrm{U} / \mathrm{ml})$ per reaction was prepared. A volume of DNA sample containing an estimated amount of up to $1 \mu \mathrm{g}$ template DNA each was added. Reactions were filled up to $50 \mu$ l total volume by addition of PCR grade water. After completion of the PCR $\left(3 \mathrm{~min}\right.$ at $95^{\circ} \mathrm{C} ; 35$ cycles of $1 \mathrm{~min}$ at $95^{\circ} \mathrm{C}, 1 \mathrm{~min}$ at $55^{\circ} \mathrm{C}, 1 \mathrm{~min}$ at $72^{\circ} \mathrm{C} ; 72^{\circ} \mathrm{C}$ for $10 \mathrm{~min}), 2 \mu \mathrm{l}$ electrophoresis buffer (6X) was added to $10 \mu \mathrm{l}$ PCR product and run at $6 \mathrm{~V} \mathrm{~cm}^{-1}$ for $30 \mathrm{~min}$ on a $1 \%$ agarose gel. When a clear band around 700 bp was observed, $40 \mu \mathrm{l}$ of the corresponding PCR product left was purified using a PCR purification kit according to its protocol. Samples were sequenced by capillary sequencing using the ITS 1 and ITS 4 primers. The two obtained sequences were aligned to a combined sequence and compared with the NCBI Nucleotide collection databases using the blast algorithm [14]. The best hit was used as an identifier for its corresponding fungal isolate.

For creation of a phylogenetic tree, established protocols were used [15]. The pre-labeled sequences were aligned to the first multiple sequence alignment (MSA) by GUIDANCE [16] using the FFT-NS-1 MAFFT algorithm [17]. A set of Neighbor-joining bootstrap trees were calculated by GUIDANCE from the first MSA $[18,19]$ followed by the creation of MSAs from the set of trees. Additional to GUIDANCE scores, Head-or-Tail (HoT) scores were calculated. Sequences with low GUIDANCE or HoT scores were excluded from the final MSA to improve the overall confidence in the final tree. MEGA5 [20] was used to identify the best suitable substitution model and distribution pattern for the final MSA through the Bayesian Information Criterion score and used subsequently to construct a hypothetic phylogenetic tree to identify promising clusters and to exclude clusters of pathogenic filamentous fungi.

\subsection{Identification of the most suitable fungus}

The potential $\beta$-1, 4-endoxylanase producers were screened in a common screening to establish a rank order sorted by activity. Subsequently, isolates were screened again independently for testing the robustness of the established rank order in comparison with the activity of a reference strain (A. niger ATCC 16888). Both screenings were done without replicates. The enzyme mixtures of the 5 fungal isolates with robust and higher or comparable $\beta$-1, 4-endoxylanase activity than the reference strain were tested regarding their activity depending on $\mathrm{pH}$ and temperature. Supernatant was prepared and $\beta$-1, 4-endoxylanase activity was measured as described earlier. Instead of using sodium acetate buffer ( $\mathrm{pH} 5)$ at a reaction temperature of $50^{\circ} \mathrm{C}$, phosphate-citrate buffer (pH 3 - 7; 1 pH step) and phosphate buffer (ph 8) were used over a broad temperature range $\left(30^{\circ} \mathrm{C}-80^{\circ} \mathrm{C} ; 10^{\circ} \mathrm{C}\right.$ steps). The absorbance values reflecting the $\beta$-1, 4-endoxylanase activity were set as percentages to the maximal measured absorbance of each isolate.

The 3 most suitable isolates regarding their $\mathrm{pH}$ and temperature dependences of $\beta$-1, 4-endoxylanase activity were compared with 4 reference strains (A. niger ATCC 16888, T. reesei ATCC 56765, T. reesei ATCC 26921 and D. dictyoides ATCC 60575) in defined xylan broth and tested for their ability to grow and produce enzymes in lignocellulose hydrolysate. For production of lignocellulose hydrolysate, empty fruit bunch powder (1 mm) was prior treated in a ratio of 1:10 with water in a Parr reactor for $10 \mathrm{~min}$ at $150^{\circ} \mathrm{C}$ as described earlier [13]. Lignocellulose hydrolysate $(40 \mathrm{ml})$ and defined xylan broth $(40 \mathrm{ml})$, respectively, were inoculated in conical flasks with spores of the isolates and the reference strains. Cultures were incubated for 8 days at $30^{\circ} \mathrm{C}$ and $200 \mathrm{rpm}$ and the $\beta$-1, 4-endoxylanase activity was measured on a regular basis. 


\section{Results and Discussion}

\subsection{Isolation and screening of $\beta-1,4$-endoxylanase producing fungi}

Totally 304 environmental samples were collected all over Singapore leading to 603 isolates of filamentous fungi. All isolates were recorded and are publically available for further usage (www.domse.org). The screening for $\beta$-1, 4-endoxylanase activity was done in 5 batches with an overall distribution of $12 \%$ active isolates, $9 \%$ possibly active isolates and $79 \%$ clearly inactive isolates. The 71 clearly active isolates were screened in a common screening to establish a rank order. YOPX-FS04 could be identified at a level of $100 \%$ as the most active isolate with all other isolates at a level of below $75 \%$.

For identification of the stability of the rank order, the isolates were screened again and all isolates at a level of below $40 \%$ were excluded from further consideration leading to 21 isolates with a clearly strong $\beta-1,4$-endoxylanase activity and a stable rank order (Fig. 1). When compared to the reference strain, the first 5 isolates were of the highest interest with 2 isolates of slightly lower activity (BBTP-FS01, YOPXFS01), 2 isolates of slightly higher activity (KRPX-FS08, WTPE-FS01) and one isolate exceeding the activity of the reference strain at almost $180 \%$ (YOPX-FS04). Therefore, all 5 isolates were selected for further experiments.

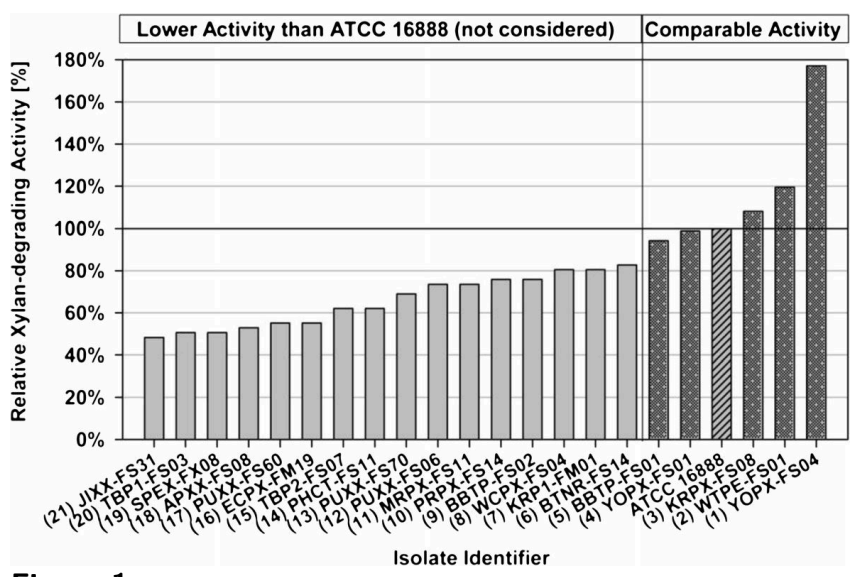

Figure 1.

Comparison of $\beta$-1,4-endoxylanase activity of the 21 most active and stable isolates with that of A. niger ATCC 16888

\subsection{Phylogenetic analysis}

For identification of clusters showing high $\beta$-1, 4-endoxylanase activity and possible pathogenic clusters which need to be reviewed more carefully before industrial usage, a phylogenetic tree based on 65 isolates (after exclusion of 6 isolates as suggested by GUIDANCE and HoT scores) was created (Fig. 2). The best fitting substitution model was determined to be a Tamura 3-parameter model following a discrete gamma distribution. The best approach was identified as a Neighbor-joining tree and the Maximum Likelihood method with a complete deletion of gaps based on 100 bootstrap replicates and a collapse of branches which could not be reproduced in at least $69 \%$ of the replicates.

Three main genera (Aspergillus, Trichoderma and Penicillium) could be identified with 32, 24 and 10 isolates, respectively. Aspergillus section nigri [21] was with 21 isolates the biggest cluster showing in average very high $\beta$-1, 4-endoxylanase activities (Fig. 3), followed by Aspergillus section flavi [22] with 8 isolates. As the section flavi is known to contain strong aflatoxin producers and human pathogens like A. flavus [23], members of this group should not be used without further investigation. Within the genus Penicillium, P. simplicissimum was with 8 isolates the largest cluster showing a similar high average activity as the Aspergilli. Even though members of the genus Trichoderma showed considerable activity levels, only T. erinaceum (5 isolates) could be considered of similarly high activity.

From the 5 most active isolates, isolate YOPX-FS01 (A.flavus/oryzae) was identified as a member of the section flavi, while the other three (YOPX-FS04, KRPXFS08, BBTP-FS01) were members of the section nigri. WTPE-FS01 was identified as P. simplicissimum.

\subsection{Identification of the most suitable filamentous fungus}

The measurement of the $\mathrm{pH}$ and temperature dependent $\beta$-1, 4-endoxylanase activity of all five isolates revealed a similar profile for YOPX-FS04, KRPX-FS08, BBTPFS01 and WTPE-FS01 with an maximal activity at $\mathrm{pH} 4$ and $60^{\circ} \mathrm{C}$ (Fig. 4a). YOPX-FS01 in opposite exhibited its maximal activity at pH 6 (Fig. 4b). Considering 


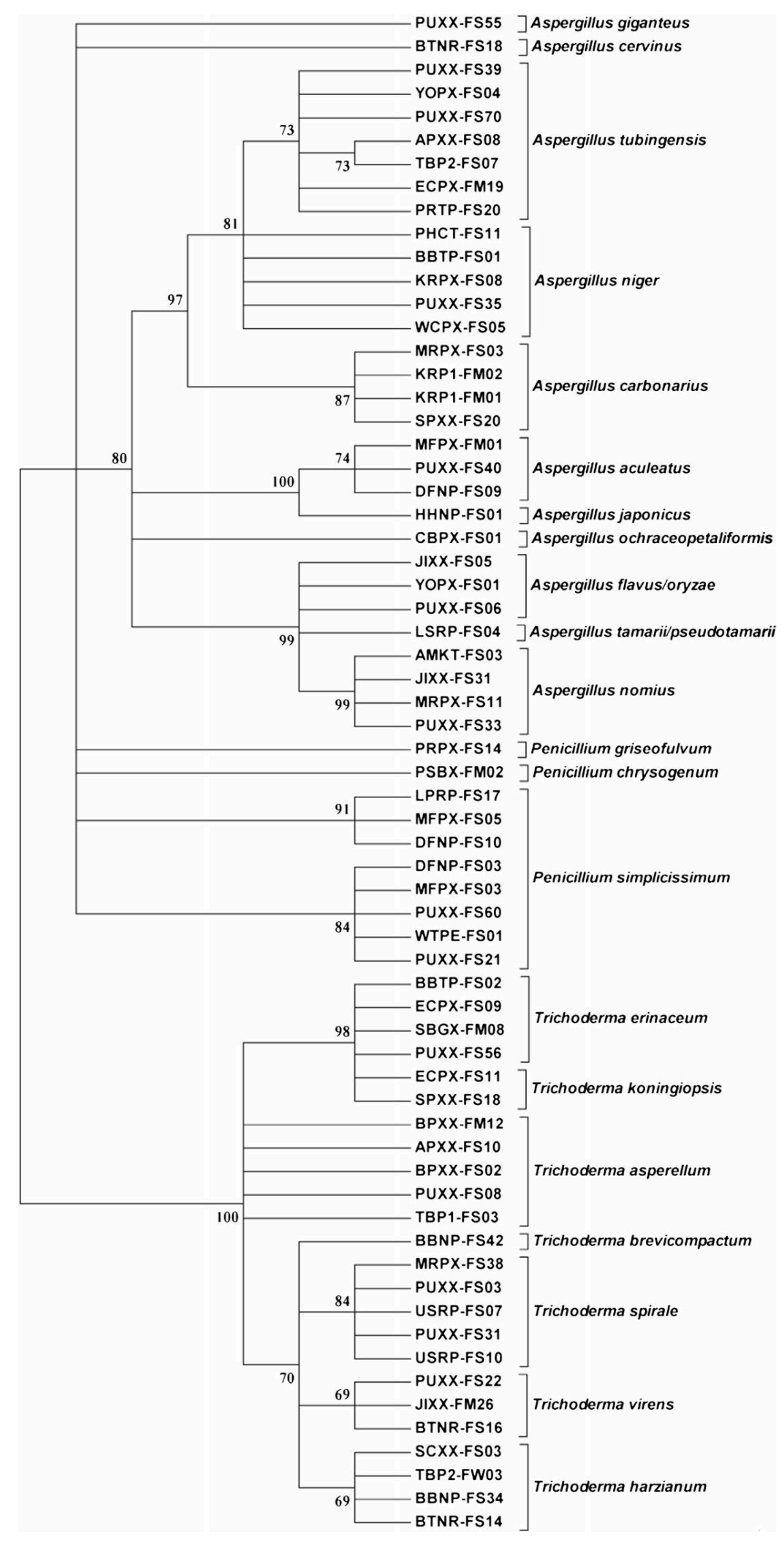

Figure 2.

Phylogenetic tree for 65 isolates exhibiting $\beta$-1,4-endoxylanase activity (numbers on branches reflect the amount of positive bootstrap replicates) 


\section{Figure 3 .}

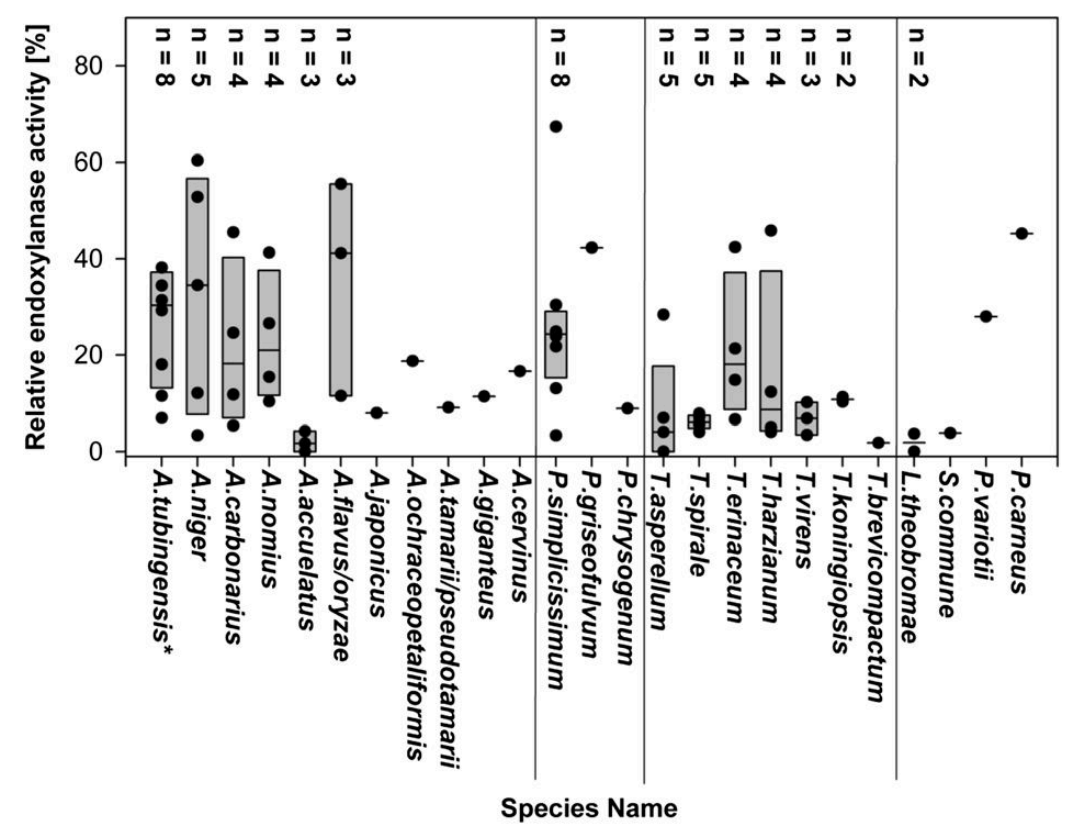

Distribution of $\beta$-1,4-endoxylanase activity among the 71 active isolates sorted on genus and species levels. The asterisk indicates that the isolate with maximal activity (100\%) is not shown
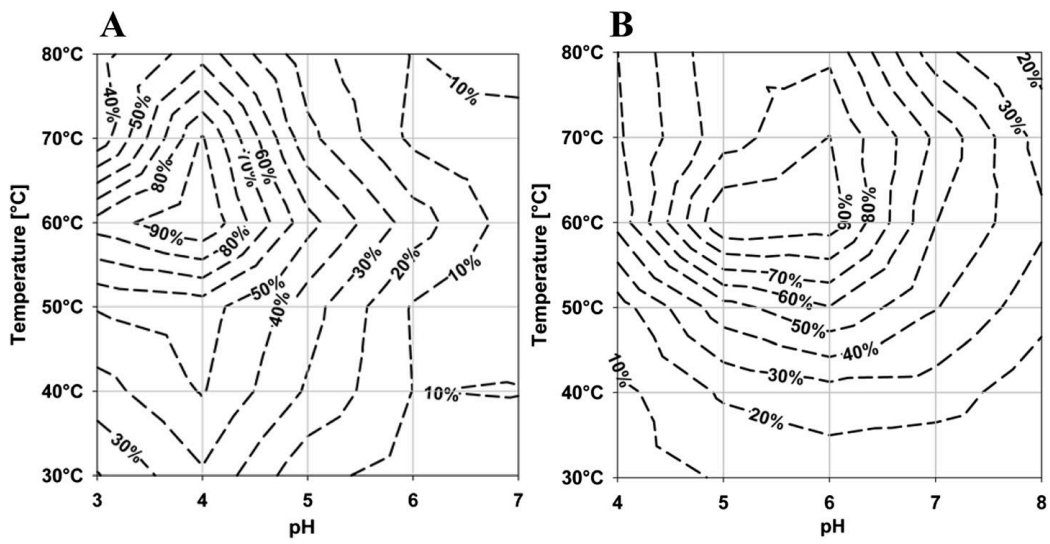

Figure 4 .

$\beta-1,4-e n d o x y l a n a s e$ activity dependence on $\mathrm{pH}$ and temperature for isolates BBTP-FS01 (a) and YOPXFS01 (b)

Figure 5.
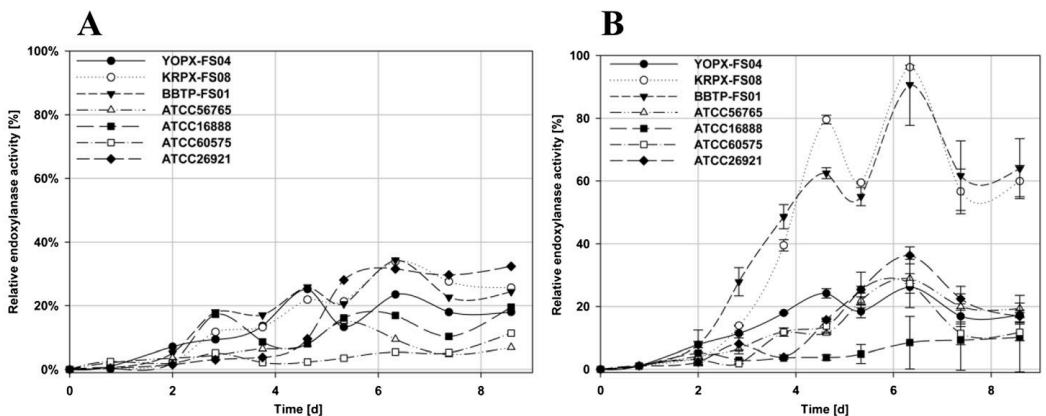

Growth of three selected isolates (YOPX-FS04, KRPX-FS08, BBTP-FS01) and four reference strains (A. niger ATCC 16888, T. reesei ATCC 56765, T. reesei ATCC 26921, and D. dictyoides ATCC 60575) on defined xylan broth (a) and lignocellulose hydrolysate (b) for 8 days (error bars represent standard deviation values) 
lactic acid production in a simultaneous saccharification and fermentation process preferably at $\mathrm{pH} 5$ and below [24], the profile of YOPX-FS01 would not be suitable. Therefore, it was excluded from further investigation as it was also found belonging to a pathogenic cluster.

After excluding WTPE-FS01 from further examinations due to poor growth, the 3 isolates left were grown together with 4 reference strains over 8 days on defined xylan broth (Fig. 5a) and lignocellulose hydrolysate (Fig. 5b), while $\beta$-1, 4-endoxylanase activity was measured in regular intervals. While no strong differences between all isolates and reference strains were observed in defined xylan media (overall activity below 40\%), $A$. niger KRPX-FS08 and $A$. niger BBTP-FS01 were found having the strongest $\beta-1$, 4 -endoxylanase activity at $100 \%$ after 6 days on lignocellulose hydrolysate while all other isolates and reference strains remained below $40 \%$ similar to the observation on defined xylan media. Isolate BBTP-FS01 was subsequently deposited as $A$. niger DSM 26641.

\section{Conclusions}

From over 600 isolates of filamentous fungi from Singaporean environment, A. niger DSM 26641 was found to be a potential and stable $\beta$-1,4-endoxylanase producer. $A$. niger DSM 26641 is capable of growing in hydrothermal lignocellulose hydrolysate exhibiting its maximal enzyme activity at $\mathrm{pH} 4$ and $60^{\circ} \mathrm{C}$. This makes the strain suitable for simultaneous saccharification and fermentation at $\mathrm{pH}$ below 5 in submerged cultures.

\section{Acknowledgments}

This work was supported by the Science and Engineering Research Council (SERC) of the Agency for Science, Technology and Research (A*STAR) under the ValueAdded Chemicals from Lignocellulose (VACL) Program (SERC grant no. 0921590133) and Biomass to Chemicals Program (SERC grant no. 1124004027) through the A*STAR Research Attachment Program (ARAP) Scholarship. We thank the National Parks Board for the permission and support to take samples within the National Parks of Singapore.

\section{References}

[1] J. Ni and G. Tokuda. Lignocellulose-degrading enzymes from termites and their symbiotic microbiota. Biotechnol. Adv., 31:838-850, 2013.

[2] P. Azadi, O. R. Inderwildi, R. Farnood, and D. A. King. Liquid fuels, hydrogen and chemicals from lignin: A critical review. Renew. Sust. Energ. Rev., 21:506-523, 2013.

[3] M. A. Abdel-Rahman, Y. Tashiro, and K. Sonomoto. Lactic acid production from lignocellulose-derived sugars using lactic acid bacteria: overview and limits. J. Biotechnol., 156:286-301, 2011.

[4] M. L. T. M. Polizeli, A. C. S. Rizzatti, R. Monti, H. F. Terenzi, J. A. Jorge, and D. S. Amorim. Xylanases from fungi: properties and industrial applications. Appl. Microbiol. Biotechnol., 67:577-91, 2005.

[5] C. Zhou, J. Bai, S. Deng, J. Wang, J. Zhu, M. Wu, and W. Wang. Cloning of a xylanase gene from Aspergillus usamii and its expression in Escherichia coli. Bioresour. Technol., 99:831-838, 2008.

[6] C. Teng, H. Jia, Q. Yan, P. Zhou, and Z. Jiang. High-level expression of extracellular secretion of a $\beta$-xylosidase gene from Paecilomyces thermophila in Escherichia coli. Bioresour. Technol., 102:1822-1830, 2011.

[7] S.-B. Zhang, H.-C. Zhai, L. Wang, and G.-H. Yu. Expression, purification and characterization of a feruloyl esterase a from Aspergillus flavus. Protein. Express. Purif., 92:36-40, 2013.

[8] H.-J. Chung, S.-M. Park, H.-R. Kim, M.-S. Yang, and D.-H. Kim. Cloning the gene encoding acetyl xylan esterase from Aspergillus ficuum and its expression in Pichia pastoris. Enzyme Microb. Technol., 31:384-391, 2002.

[9] J. S. Van Dyk and B. I. Pletschke. A review of lignocellulose bioconversion using enzymatic hydrolysis and synergistic cooperation between enzymes factors affecting enzymes, conversion and synergy. Biotechnol. Adv., 30:1458-1480, 2012.

[10] R. Krull, T. Wucherpfennig, M. E. Esfandabadi, R. Walisko, G. Melzer, D. C. Hempel, Kwade A. 
Kampen, I., and C. Wittmann. Characterization and control of fungal morphology for improved production performance in biotechnology. J. Biotech., 163:112-123, 2013.

[11] C. M. P. Braga, P. da Silva Delabona, D. J. da Silva Lima, D. A. A. Paixão, J. G. da Cruz Pradella, and C. S. Farinas. Addition of feruloyl esterase and xylanase produced on-site improves sugarcane bagasse hydrolysis. Bioresour. Technol., 170:316-324, 2014.

[12] P. Biely, R. Mislovicova, and D. Toman. Remazol brilliant blue-xylan: a soluble chromogenic substrate for xylanases. Methods Enzymology, 160:536-541, 1988.

[13] C. Ottenheim, C. Verdejo, W. Zimmermann, and J. C. Wu. Hemicellulase production by Aspergillus niger dsm 26641 in hydrothermal palm oil empty fruit bunch hydrolysate and transcriptome analysis. J. Biosci. Bioeng., 118:696-701, 2014.

[14] S. F. Altschul, T. L. Madden, A. A. Schäffer, J. Zhang, Z. Zhang, W. Miller, and D. J. Lipman. Gapped blast and psi-blast: a new generation of protein database search programs. Nucleic Acids Res., 25:3389-3402, 1997.

[15] B. G. Hall. Building phylogenetic trees from molecular data with mega. Mol. Biol. Evol., 30:1229-1235, 2013.

[16] O. Penn, E. Rivman, H. Ashkenazy, G. Landan, D. Graur, and T. Pupko. Guidance: a web server for assessing alignment confidence scores. Nucleic Acids Res., 38:W23-W28, 2010.

[17] K. Katoh, K. Kuma, H. Toh, and T. Miyata. Mafft version 5: improvement in accuracy of multiple sequence alignment. Nucleic Acids Res., 33:511-518, 2005.

[18] N. Saitou and M. Nei. The neighbor-joining method: a new method for reconstructing phylogenetic trees. Mol. Biol. Evol., 4:406-425, 1987.

[19] J. Felsenstein. Confidence limits on phylogenies: an approach using the bootstrap. Evolution, 39:783-791, 1985.
[20] K. Tamura, D. Peterson, N. Peterson, G. Stecher, M. Nei, and S. Kumar. Mega5: molecular evolutionary genetics analysis using maximum likelihood, evolutionary distance, and maximum parsimony methods. Mol. Biol. Evol., 28:2731-2739, 2011.

[21] R. A. Samson, P. Noonim, M. Meijer, J. Houbraken, J. C. Frisvad, and J. Varga. Diagnostic tools to identify black aspergilli. Stud. Mycol., 59:129-145, 2007.

[22] L. Wang, K. Yokoyama, H. Takahasi, N. Kase, Y. Hanya, K. Yashiro, M. Miyaji, and K. Nishimura. Wang, l. and yokoyama, k. and takahasi, h. and kase, n. and hanya, y. and yashiro, k. and miyaji, m. and nishimura, k. Int. J. Food Microbiol., 71:75-86, 2001.

[23] M. T. Hedayati, A. C. Pasqualotto, P. A. Warn, P. Bowyer, and D. W. Denning. Aspergillus flavus: human pathogen, allergen and mycotoxin producer. Microbiology, 153:1677-1692, 2007.

[24] M. Hongo, Y. Nomura, and M. Iwahara. Novel method of lactic acid production by electrodialysis fermentation. Appl. Microbiol. Biotechnol., 52:314-319, 1986. 